\title{
Towards Accessible Mental Healthcare through Augmented Reality and Self-Assessment Tools
}

\author{
https://doi.org/10.3991/ijoe.v16i04.12095 \\ Victoria Lush $\left({ }^{凶}\right)$, Christopher Buckingham \\ Aston University, Birmingham, UK \\ v.lushleaston.ac.uk \\ Suzanne Edwards \\ Enlighten, Tamworth, UK \\ Ulysses Bernardet \\ Aston University, Birmingham, UK
}

\begin{abstract}
Mental health presents a growing public health concern worldwide with mental illnesses affecting people's quality of life and causing an economic impact on societies. The rapidly increasing demand for mental healthcare is calling for new ways of disseminating mental health knowledge and for supporting people with mental health illnesses. As an alternative to traditional mental health therapies and treatments, mental health self-assessment and self-management tools become widely available to the public. While such tools can potentially offer more timely personalised support, individuals seeking help are faced with the challenge of making an appropriate choice from an exhaustive number of online tools, mobile apps, and support programs.

In this article, we present myGRaCE - a self-assessment and self-management mental health tool made accessible to users via Augmented Reality technologies. The advantage of the system is that it provides a direct pathway to relevant and reliable mental health resources and offers a positive incentive and interventions for at-risk users. To investigate the usability and intuitiveness of the system, we conducted a pilot evaluation study with 10 participants. The results showed that the majority of study participants found the system intuitive and easy to use.
\end{abstract}

Keywords-Mental health, mental health disorders, self-assessment tools, selfmanagement, Augmented Reality.

\section{Introduction}

Worldwide mental health illnesses are increasingly impacting not only peoples' quality of living but also national economies. Global statistics show that in 2013, depression was the second global leading cause of disability after back pain and the primary factor leading to disability in 26 counties [1]. In 2017, nearly 4 per cent of people were affected by depression and anxiety disorders worldwide [2]. 
Suicide being identified as the second leading cause of death among 15-29 year-olds globally in 2016 means that close to 800,000 people die due to suicide every year [3]. While not all suicides and suicide attempts are directly caused by underlying mental health disorders, it is estimated that nearly $85 \%$ of suicides in North America, Western Europe and Australia and 68\% in China, India and Taiwan are attributable to mental and substance use disorders [4]. Studies further suggest that the risk of suicide is approximately 20 times higher for individuals with depression [1].

Mental ill-health is wide-ranging with different symptoms and severity and is generally characterised by abnormal thoughts, emotions, behaviour, relationships with others, or a combination of these [5]. Common examples of such disorders include anxiety, panic attacks, depression, schizophrenia, phobias, personality disorders, obsessivecompulsive disorder (OCD), and post-traumatic stress disorder (PTSD) [5]. Mental health disorders are universal and can affect individuals of all ages and gender, from different backgrounds, societies and environment, with different levels of income and quality of life.

While many mental health disorders and illnesses can be successfully treated, or at least managed, early intervention is crucial to make the difference between successful recovery and relapse to years of disability [6]. Unfortunately, in the countries where mental health is highly stigmatised or is seen as very taboo, public mental healthcare is not available. In the countries where public mental healthcare schemes exist, the services are often thinly stretched, with budget increases too small for the demand, shortage of staff, and a lack of resources to sustain timely and quality support. To tackle the rapidly increasing demand for mental healthcare, novel, accessible ways of disseminating mental health knowledge and supporting people with mental health illnesses are needed.

Our goal is to develop an accessible interactive mental health support tool that will offer a direct pathway to relevant information; help service users to understand, assess, monitor, and manage their mental health; and provide appropriate interventions or access to professional help when needed. In this article, we present a system that combines myGRaCE self-assessment and self-management mental health tool with Augmented Reality technology to provide mental health support in an accessible and easy to use way. The system offers service users the resources to increase control of their mental health conditions via self-monitoring and self-help.

\section{Self-Assessment and Self-Management Tools}

Self-assessment and self-management tools become common alternatives to traditional mental health therapies and treatments, offering a more cost-effective way to enable service users to self-assess their mental health, identify the need for clinical intervention, or manage their condition via self-help [7]. Such tools can also help improve mental health literacy which can, in turn, facilitate early recognition, help-seeking, and timely appropriate intervention [8]. 
Self-assessment screening tools provide the facilities to determine whether individuals are experiencing symptoms of mental health conditions without having direct access to the therapist [9]. These tools are typically delivered in a form of free and anonymous online questionnaires that address a particular mental health condition (e.g., bipolar disorder, depression, PTSD).

Self-management tools can be delivered as training courses for the affected individuals or carers, self-help groups with mutual and/or peer support, online or paper-based personal health and wellbeing recovery plans designed for the individuals or carers, or mobile apps that offer real-time and 'on-the-go' support and therapy. Self-management tools help to put individuals in direct control of their condition by enabling them to continuously monitor the progress of their recovery, cope by themselves, and respond to the indicators of deteriorating health before seeking clinical interventions [10].

While self-assessment and self-management tools can potentially offer more timely personalised support, individuals seeking help are faced with the challenge of making an appropriate choice from an exhaustive number of online questionnaires, mobile apps, and support programs.

\section{MyGRaCE Self-Assessment Tool}

GRiST (Figure 1) is a web-based decision support system designed for mental health practitioners to enable the assessments and management of risks associated with mental health problems. GRiST has been developed for over 17 years and it is based on real expert mental health practitioners' knowledge. The unique nature of GRiST is in that it integrates real patient data, mental-health practitioners' expertise and empirical evidence to support comprehensive clinical risk evaluations, risk formulations, and safety plans. It bases its expertise on a psychological model of classification because this improves intuitive understanding of how the system works. The underlying knowledge hierarchy is derived from the way expert mental health practitioners think and reason about mental health and is used to drive the system functionality [11].

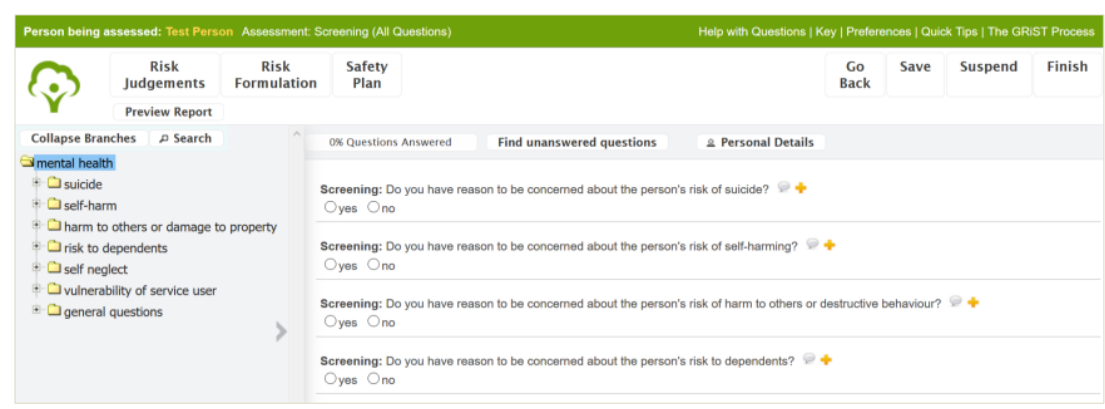

Fig. 1. GRiST mental health decision support tool (https://www.egrist.org/)

Assessors (e.g., mental health practitioners or nurses) can use GRiST to collect a comprehensive set of data about service users that they then associate with clinical risk 
judgements for suicide, self-harm, harm to others, self-neglect, and vulnerability. The formal specification of GRiST knowledge facilitates flexible interfaces that ask the questions in different ways and in different orders depending on the type of service user and the context of assessment.

The self-assessment version of GRiST, myGRaCE (Figure 2), converts the clinical questions into ones that are more suitable for asking of oneself. The flexible structure and validated questions of GRiST can thus be used to engage the service user in realistic self-dialogues when assessing themselves.

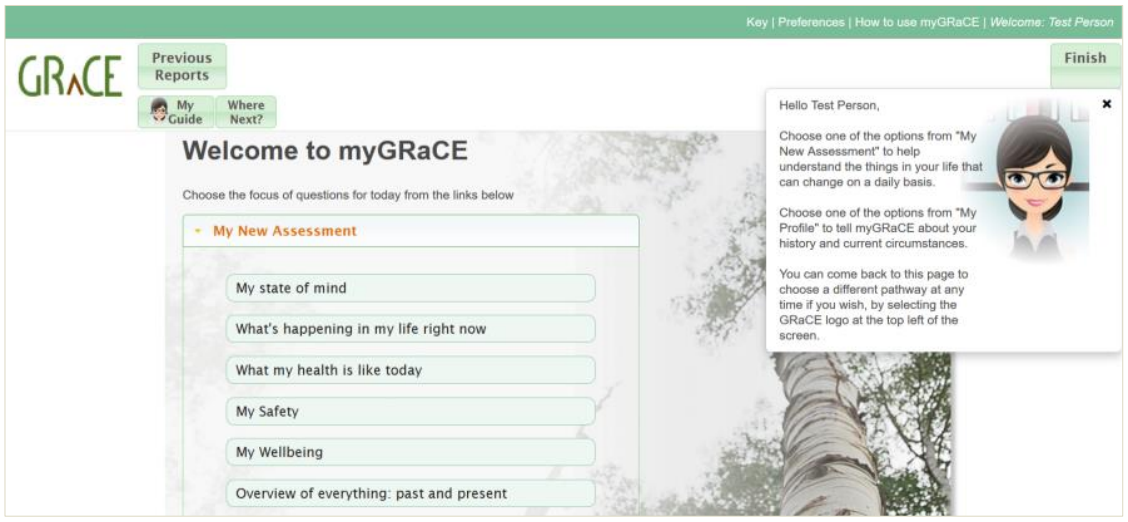

Fig. 2. MyGRaCE self-assessment version of GRiST tool

An important caveat for any type of online therapy is that it can increase risks if badly conducted. GRiST can help mitigate this because it has a database of 250,000 clinical risk judgements ranging from zero (minimal or normal risk) to 10 (maximum risk) given by several thousand expert mental-health practitioners. Mathematical algorithms are able to simulate the risk judgements mental-health practitioners would give for data collected through a self-assessment using myGRaCE. Interventions built into the GRiST, and consequently into myGRaCE, knowledgebase can trigger appropriate support if the risks go above certain thresholds. The underlying hierarchical knowledgebase enables the same algorithms to explain which parts of the assessment are most influential on the risks.

\section{$4 \quad$ Augmented Reality for Mental Health}

Augmented Reality (AR) is an interactive technology that integrates digital content into the user's physical environment [12]. Unlike Virtual Reality (VR), which places the user in a simulated artificial environment, AR allows "augmentation" of real user experience by enhancing it with visual, sound, tactile, olfactory, and gustatory information [13]. AR and VR technologies are not just for entertainment or gaming, they have a real potential to make a difference in the healthcare industry and, in mental 
healthcare, they can become an effective solution for timely interventions and stimulation of personal change in people with mental health illnesses [14].

Enlighten is a UK enterprise that provides AR resources for mental health services and commissioners. Enlighten produced the world's first AR campaign for Rethink Mental Illness - the largest mental health charity in Europe, raising mental health awareness through AR resources. The campaign is delivered via AR posters (Figure 3) that facilitate easy and informal access to short Rethink mental health videos and links to further support areas, rather than the users having to search for relevant information on the website. Interactive AR resources are activated by pointing a mobile device at a unique tag embedded in the poster content which makes the poster "come alive". The project helps to overcome stigma and the lack of knowledge about mental health.

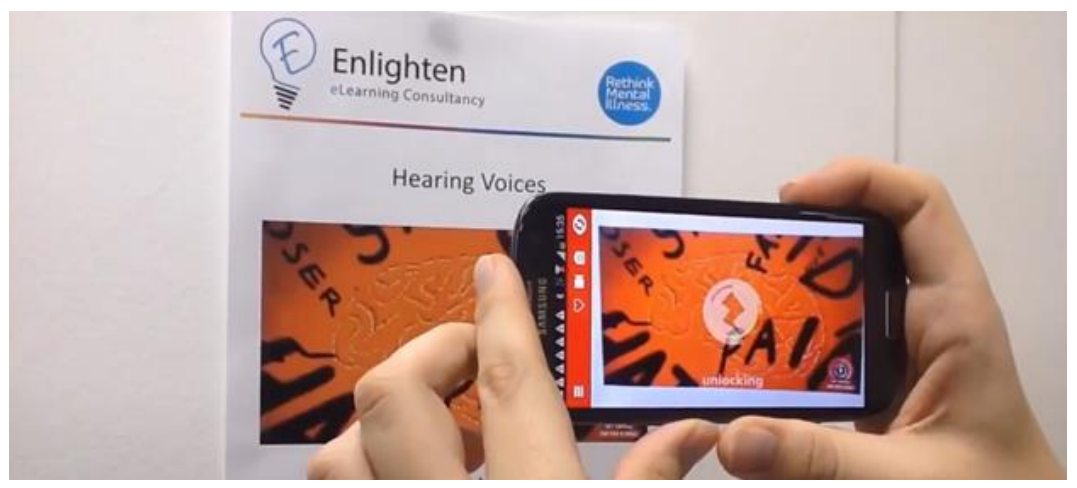

Fig. 3. Accessing AR content via augmented poster

Mental health AR posters are currently being used in over 200 establishments across the UK including schools, colleges, universities, businesses, NHS trusts, community centres, local authority buildings, and training providers. Based on the campaigns that targeted various educational establishments, to date, there has been a 59\% increase in self-referrals to counselling and mental wellbeing services. A significant number of those referrals related to self-harm (42\% increase), suicidal tendencies, threats or attempts ( $8 \%$ increase), and diagnosed mental health concerns (58\% increase).

Building on the success of the mental health AR campaigns, our goal is to link those who need mental health support with myGRaCE self-help tool using AR technology.

\section{$5 \quad$ Augmented Reality Mental Health Support System}

The developed AR mental health support system combines the interactive AR resources with myGRaCE self-help tool. The system framework is shown in Figure 4 and comprises three main components: a mobile device that has the "Zappar" app installed; physical objects tagged with unique "zapcodes" (e.g., posters, leaflets, key rings); and sets of interactive mental health resources linked to the zapcodes (including access to myGRaCE tool). 
The AR part of the system is built using the Zappar platform that allows creating, managing and distributing AR content on mobile devices. Zapcodes (which are similar to barcodes and QR codes) are used to uniquely identify sets of resources, e.g., a set of animations, videos, text, weblinks, and geolocation-based content. Each unique zapcode can provide access to a combination of resources covering a specific mental health topic such as anxiety, panic attacks, PTSD, suicide, and many others. Zapcodes can be placed on various physical objects such as printed posters, leaflets, key rings, or sprayed on a pavement. The latter is particularly useful for positioning codes in an unobtrusive way. To access the content linked to the zapcode, the service user is required to activate the Zappar app and point the mobile device camera at the tagged item. The Zappar app will then display the AR content corresponding to the zapcode marker as well as to the context of the user's action.

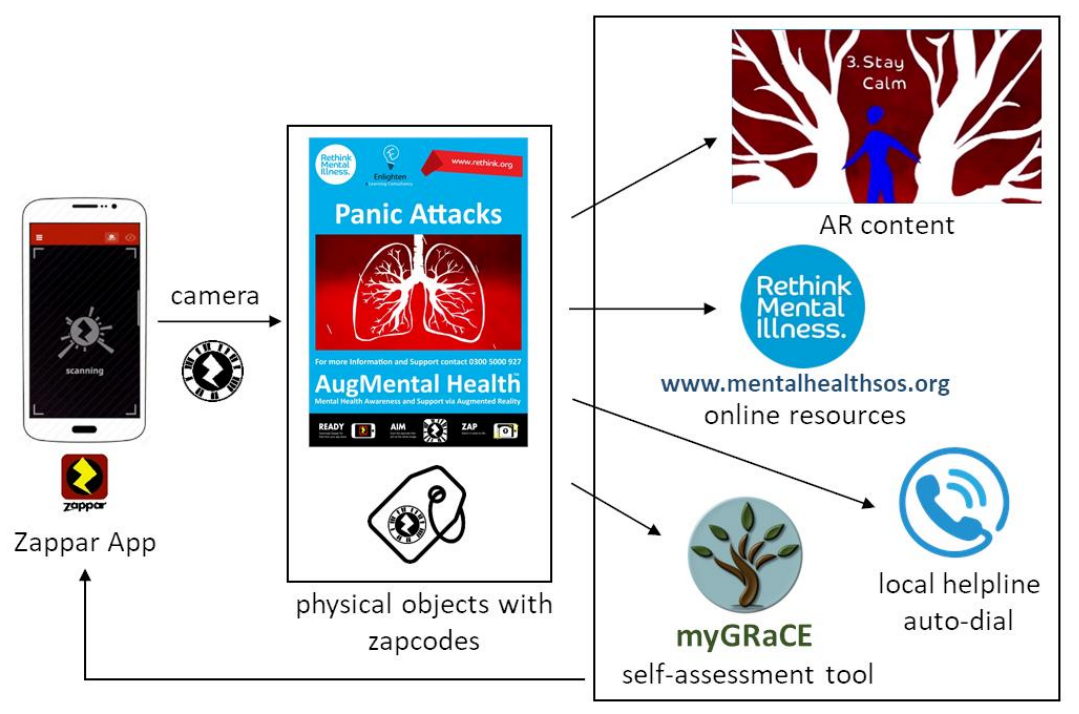

Fig. 4. Framework of the Augmented Reality mental health support system

In addition to the interactive AR content described above, the system provides a direct link to a mobile version of myGRaCE self-assessment tool (Figure 5). Here, myGRaCE offers several mental wellbeing topics (e.g., "My life right now") that the service user can select to complete the self-assessment. 


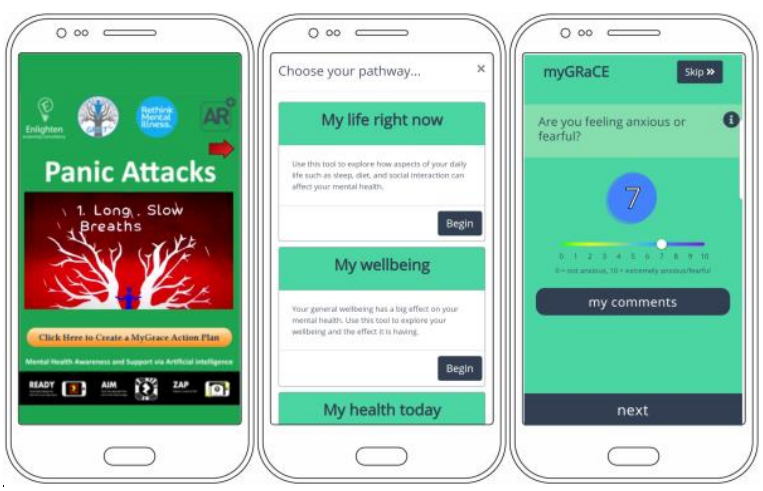

Fig. 5. Left: AR system home screen; middle: myGRaCE; right: self-assessment question

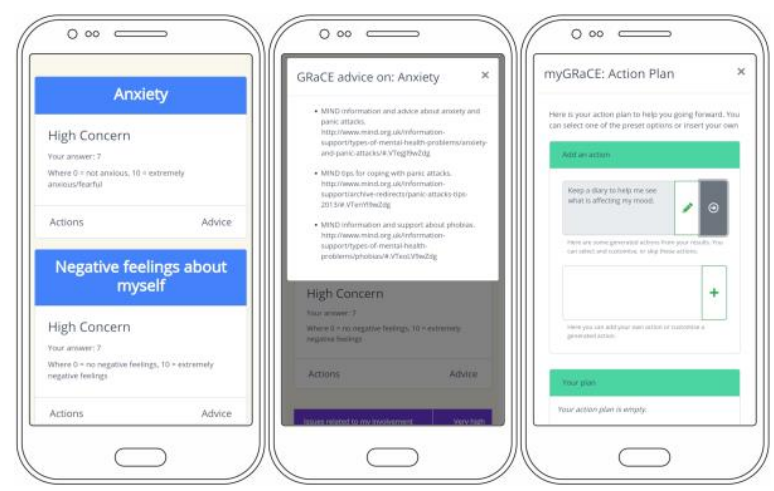

Fig. 6. Left: self-assessment summary; middle: sample advice; right: self-help plan

Once the user completes the self-assessment, myGRaCE provides the assessment summary helping the user to gain a better understanding of his/her mental state and highlighting any areas of concern (Figure 6). For each area with a high level of concern, myGRaCE offers relevant advice and access to further resources. In addition to the recommendations, the tool encourages the user to create a self-monitoring and self-help action plan to further increase control of the user's mental health conditions.

\section{Evaluation Study}

To investigate the usability and intuitiveness of the AR mental health support system, we conducted a pilot evaluation study with 10 Computer Science department staff members and $\mathrm{PhD}$ students (6 male, average age 31). All participants were familiar with the term Augmented Reality, however, only 5 have previously used AR systems.

During the study, participants were presented with four mental health AR posters (Stress, Anxiety, Panic Attacks, and PTSD) placed on the wall of the study room. Only the Panic Attacks poster included myGRaCE self-assessment tool. Participants were asked to consider a scenario where they encountered these posters at their workplace 
and decided to learn their content. Participants were asked to think-aloud while interacting with the posters and were given prompts if they were unable to proceed further. Once participants identified the need for a mobile app, they were provided with a Samsung S7 mobile phone with the Zappar app installed. After the interaction, participants were asked to complete a 7-scale Likert questionnaire and describe what they liked the most about the system, what they liked the least, and what they would improve.

The study session lasted up to 30 minutes and participants spent on average 20 minutes interacting with the system. Out of 10 participants, 6 identified that they required an app for AR content and 5 accessed the myGRaCE tool without a prompt.

Post-study questionnaire results (Table 1) showed that on average participants found the system intuitive and easy to use. The organisation of information was clear and various functions were well integrated. Participants felt confident using the system and thought that many people would learn how to use the system very quickly. Participants were however unsure whether they would use this system frequently.

Table 1. Post-study questionnaire results ( 1 - strongly agree; 4 - neutral; 7 - strongly disagree)

\begin{tabular}{|l|c|}
\hline \multicolumn{1}{|c|}{ Post-study questions } & Average \\
\hline I found the system intuitive & 2.3 \\
\hline I found the system easy to use & 2.2 \\
\hline I found the system unnecessary complex & 5.4 \\
\hline I think I would need support to use this system & 5.6 \\
\hline I found the various functions in the system well integrated & 2.6 \\
\hline I think many people would learn how to use the system very quickly & 2.1 \\
\hline I found the system very cumbersome/awkward to use & 5.6 \\
\hline I felt very confident using the system & 2.5 \\
\hline I think I would like to use this system frequently & 3.4 \\
\hline The organisation of information in the system was clear & 2.7 \\
\hline
\end{tabular}

Post-study interviews revealed that all participants liked the interactivity and AR capability of the system. Participants found the content and self-assessment tool useful and relevant. Regarding the system improvements, participants commented that the instruction for activating AR content should be more explicit. It was also suggested to introduce a short tutorial to explain different content and improve information flow. All participants agreed that, while the video content is engaging, the playback should not start automatically and videos should be shorter not to distract from other content.

\section{Conclusion}

In this paper, we introduced a mental health support system that combines an information resource - the myGRaCE self-assessment/self-management tool - with AR technology. The key advantages of this system are an easily accessible AR interface, a reliable self-assessment service based on real practitioners' knowledge, the direct pathway to relevant Rethink Mental Illness resources, and the provision of personalised support and interventions. 
We also presented the initial results from a pilot evaluation study that was conducted to investigate system usability and intuitiveness. The next stage of our research will focus on applying the essential improvements identified through the study and deploying the system at various establishments (e.g., universities, community centres) to collect formal feedback from potential service users.

\section{$8 \quad$ References}

[1] Ferrari, A.J., Charlson, F.J., Norman, R.E., Patten, S.B., Freedman, G., Murray, C.J.L., and Whiteford, H.A. (2013). Burden of depressive disorders by country, sex, age, and year: Findings from the Global Burden of Disease study 2010. PLOS Medicine, 10:11. https://doi.org/10.1371/journal.pmed.1001547

[2] Ritchie, H. and Roser, M. (2018). Our World in Data: Mental Health. Available at: https://ourworldindata.org/mental-health\#anxiety-disorders. Accessed on 03 February 2020.

[3] WHO (2018). Suicide. Available at: https://www.who.int/news-room/fact-sheets/detail/suicide. Accessed on 03 February 2020.

[4] Ferrari, A.J., Norman, R.E., Freedman, G., Baxter, A.J., Pirkis, J.E., and Harris, M.G. (2014). The burden attributable to mental and substance use disorders as risk factors for suicide: Findings from the Global Burden of Disease Study 2010. PLoS ONE, 9(4): e91936. https://doi.org/10.1371/journal.pone.0091936

[5] WHO (2019). Mental Disorders. Available at: https://www.who.int/mental_health/management/en/. Accessed on 03 February 2020.

[6] Yu, H., Zhang, G., Liu, J. and Li, K. (2019). Intelligent knowledge service system based on depression monitoring of college students. International Journal of Emerging Technologies in Learning, 14(12):71-84. https://doi.org/10.3991/ijet.v14i12.10702

[7] Stathopoulou, A., Karabatzaki, Z., Kokkalia, G., Dimitriou, E., Loukeri, P.I., Economou, A., and Drigas, A. (2018). Mobile assessment procedures for mental health and literacy skills in education. International Journal of Interactive Mobile Technologies (iJIM), 12(3):21-37. https://doi.org/10.3991/ijim.v12i3.8038

[8] Karasouli, E. and Adams, A. (2014). Assessing the evidence for e-resources for mental health self-management: a systematic literature review. JMIR Mental Health, 1(1):e3. https://doi.org/10.2196/mental.3708

[9] Donker, T., Petrie, K., Proudfoot, J., Clarke, J., Birch, M.R., and Christensen, H. (2013). Smartphones for smarter delivery of mental health programs: A systematic review. Journal of Medical Internet Research, 15(11):e247. https://doi.org/10.2196/jmir.2791

[10] Lintvedt, O.K., Griffiths, K.M., Sørensen, K., Østvik, A.R., Wang, C.E., Eisemann, M., and Waterloo, K. (2013). Evaluating the effectiveness and efficacy of unguided internet-based self-help intervention for the prevention of depression: A randomized controlled trial. Clinical Psychology \& Psychotherapy, 20(1):10-27. https://doi.org/10.1002/cpp.770

[11] Buckingham, C.D., Adams, A., and Mace, C. (2008). Cues and knowledge structures used by mental-health professionals when making risk assessments. Journal of Mental Health, 17(3):299-314. https://doi.org/10.1080/09638230701498374

[12] Caudell, T.P. and Mizell, D.W. (1992). Augmented reality: An application of heads-up display technology to manual manufacturing processes. In Proceedings of the Twenty-Fifth Hawaii International Conference on System Sciences, 2:659-669. https://doi.org/10.1 $\underline{109 / \text { hicss.1992.183317 }}$ 
[13] Boonbrahm, P., Kaewrat, C., Pengkaew, P., Boonbrahm, S., and Meni, V. (2018). Study of the hand anatomy using real hand and augmented reality. International Journal of Interactive Mobile Technologies (iJIM), 12(7):181-190. https://doi.org/10.3991/ijim.v12i7.9645

[14] Dar, S., Lush, V. and Bernardet, U. (2019). The Virtual Human Breathing Relaxation System. In Proceedings of the 5th Experiment International Conference (exp. at'19), 276-277. https://doi.org/10.1109/expat.2019.8876478

\section{Authors}

Victoria Lush is a Research Fellow in Computer Science at Aston University, Aston Triangle, Birmingham, B4 7ET, UK (email: v.lush1@aston.ac.uk).

Christopher Buckingham is a Reader in Computer Science at Aston University, Aston Triangle, Birmingham, B4 7ET, UK.

Suzanne Edwards is the Head of Education and Qualification Design at Enlighten, Tamworth Enterprise Centre, Philip Dix House, Tamworth, B79 7DN, UK.

Ulysses Bernardet is a Lecturer in Computer Science at Aston University, Aston Triangle, Birmingham, B4 7ET, UK.

Article submitted 2019-10-28. Resubmitted 2020-02-03. Final acceptance 2020-02-03. Final version published as submitted by the authors. 